Journal of the Society of Rheology, Japan Vol.31, No.4, $219 \sim 227$ (2003)

(Nihon Reoroji Gakkaishi)

Article

(C)2003 The Society of Rheology, Japan

\title{
Viscoelasticity of Aluminium Neutralized Telechelic Poly(ethylene butylene) lonomer
}

\author{
Syed Atiqur RAHMAN, and Norio NemOTO* \\ Department of Molecular and Material Sciences, IGSES, Kyushu University, \\ Hakozaki, Fukuoka 812-8581, Japan \\ (Received : April 21, 2003)
}

\begin{abstract}
The associative and mechanical properties of aluminium neutralized telechelic poly(ethylene butylene) ionomer, CTPEB-Al, in a nonpolar solvent of decalin were studied from very dilute solution up to the bulk with rheological methods and IR spectroscopy. Dilute solution viscometry revealed that CTPEB-Al molecules are associated in very dilute solution as their smallest associating structural unit made of three CTPEB chains and two aluminium tricarboxylate bonds $\left[\left(\mathrm{COO}^{-}\right)_{3} \mathrm{Al}^{3+}\right.$ : ATC bond]. These smallest associating units are further associated into larger aggregates with increasing polymer concentration $C$ and form viscoelastic networks above $C=7 \mathrm{wt} \%$. The viscoelasticity and IR data reveal that in the low concentration range from $7 \mathrm{wt} \%$ to $12 \mathrm{wt} \%$, CTPEB-Al chains are associated with each other in such a way that they form pretty long network strands with or without branches as dangling chains that act as elastically effective chains as a whole. In the networks with $C \geq 70 \mathrm{wt} \%$, however, most of the ATC bonds and ion clusters act as effective crosslinking points so that almost $90 \%$ chains become elastically effective in the bulk state.
\end{abstract}

Key Words: Viscoelasticity / Telechelic ionomer / Aluminum neutralization / CTPEB / Network structure / Ion cluster

\section{INTRODUCTION}

Ionomers are polymers containing small amount of ionic groups onto the nonionic backbone chains which have a wide range of industrial application. ${ }^{1)-4)}$ Telechelic ionomers that contain ionic groups only at chain ends can act as model system to study association behaviors of ionomers more clearly and deeply. Many experimental studies have been performed separately on both the bulk and solution state of ionomers to understand the structure and associating behavior of ionomer in recent years. ${ }^{5-40)}$ But so far known to us, there is no study which has dealt with the association process of ionomers from very dilute solution state up to the bulk. Telechelic ionomers may be considered as the best sample for this purpose.

In previous reports, ${ }^{40,41)}$ we studied in detail the viscoelasticity of potassium and magnesium neutralized telechelic poly (ethylene butylene) ionomers (CTPEB-K and CTPEB-Mg) in a nonpolar solvent of decalin and also in bulk. For CTPEB-K we found that the chains are associated with each other in a pairwise manner by association of ionic groups at chain ends so as to form linear chain-like aggregates even in the very

* To whom correspondence should be addressed.

E-mail: nemo@mm.kyushu-u.ac.jp dilution limit. With increasing concentration $C$, CTPEB-K formed huge polymeric clusters in the system and further increase in $C$ gave rise to a viscoelastic network and to the stronger network as concentration further increased. For CTPEB-Mg we found that the chains are associated as ring polymer in very dilute solution of decalin, followed by linear association of rings with increasing $C$. The linearly associated rings form huge polymeric clusters of different sizes as $C$ further increased and a weak viscoelastic network is formed near the overlapping concentration $C^{*}$ of CTPEB-Mg chains. The network becomes stronger as $C$ further increases. However, the CTPEB-K network formed at $C$ lower than the corresponding $C$ for the CTPEB-Mg network is more elastic than CTPEB-Mg at the same $C$. An appreciable difference in the formation mechanism of the viscoelastic network between CTPEB-K and CTPEB-Mg systems has led us to that similar studies should be performed using CTPEB samples neutralized with a trivalent or tetravalent cation at chain ends to elucidate the valence effects on association processes and also evolution of viscoelastic networks due to the formation of ionic clusters with an increase in $C$.

In this work we performed rheology and IR spectroscopy measurements on aluminium neutralized telechelic poly (ethylene butylene) ionomer (CTPEB-Al) in decalin over the 
whole concentration range from the very dilute $C$ region to $100 \mathrm{wt} \%$. We found that most of CTPEB-Al molecules are associated in very dilute decalin solution as their smallest associating structural unit made of three CTPEB chains and two aluminium tricarboxylate bonds (ATC bonds). With increasing $C$, association among these smallest associating unit may proceed through dipole-dipole attraction between ATC bonds so that different sizes of polymeric clusters are formed in solutions up to $C=6 \mathrm{wt} \%$. When these polymeric clusters come very close to each other at $C=7 \mathrm{wt} \%$, a weak viscoelastic network is formed with a very small number of elastically effective strands. The network becomes stronger with increasing $C$ due to an increase in the number of elastically effective strands, resulting in that almost $90 \%$ chains are found elastically effective in the bulk.

\section{EXPERIMENTAL}

\subsection{Materials}

CTPEB-Al and esterified CTPEB (CTPEB-Ester) samples were prepared from carboxyl terminated poly (ethylene butylene) copolymer (CTPEB) with the number-average molecular weight of $M_{\mathrm{n}}=1400\left(M_{\mathrm{w}} / M_{\mathrm{n}} \approx 1.2\right.$, functionality = 2.15, Nippon Soda Co. Ltd). CTPEB-Ester was prepared as ethyl carboxylate with the acylchloride method as described elswhere. ${ }^{40)}$

CTPEB-Al was prepared by complete neutralization of dried CTPEB with stoichiometric amount of aluminium ethoxide. Prescribed amounts of CTPEB and aluminium ethoxide were dissolved in toluene and in a mixture of thionyl chloride and tetrahydrofuran (THF) with a ratio of 5 to 1 , respectively. Neutralization was carried out in a distillation apparatus at $60^{\circ} \mathrm{C}$ under the vacuum condition to evaporate ethanol and solvents. The sample was once again vacuum dried for several days at $80^{\circ} \mathrm{C}$ until all solvents were removed. The absorption peak of the carboxylic acid group completely disappeared, while new absorption peaks at $1645 \mathrm{~cm}^{-1}$ and $1585 \mathrm{~cm}^{-1}$ to be assigned to the carboxylate group appeared.

Anhydrous decalin was used as solvent for preparation of CTPEB-Ester and CTPEB-Al solutions with polymer concentration $C$ from the very dilute one to the bulk. Weighed amounts of CTPEB-Al and the solvent were put into a vial and kept for at least two days so as to penetrate the solvent into CTPEB-Al. Then the vial was rotated at $0.2 \mathrm{rpm}$ for about $12 \mathrm{~h}$ at $80^{\circ} \mathrm{C}$ to make samples homogeneous and transparent.

\subsection{Methods}

Viscosities of dilute solutions for CTPEB-Al and CTPEBEster in decalin were measured with an Ubbelohde type of capillary viscometer at $25 \pm 0.05^{\circ} \mathrm{C}$. Rheological measurements were performed using a stress-controlled rheometer (CarriMED CSL-100, UK) with a cone-and-plate type geometry of diameter $4 \mathrm{~cm}$. A solvent trap equipped with the rheometer was used to protect the sample from evaporation. The sample chamber was then covered with an external round heater box made of epoxy resin to minimize the temperature gradient in the sample.

To carry out the viscoelastic measurements of CTPEB-Al solutions with $C$ in the range of $7 \sim 12 \mathrm{wt} \%$, the samples were heated to obtain a viscous liquid and then transferred to the flat surface of the rheometer whose temperature was set at about $60^{\circ} \mathrm{C}$, and then temperature was decreased to $15^{\circ} \mathrm{C}$. This procedure was useful to prevent formation of bubbles inside the samples and also to avoid rupture of the samples. The storage and the loss shear moduli, $G^{\prime}(\omega)$ and $G^{\prime \prime}(\omega)$, were measured in a range of angular frequency $\omega$ from 0.01 to 100 $\operatorname{rads}^{-1}$ at five temperatures from 15 to $55^{\circ} \mathrm{C}$. For $70 \mathrm{wt} \%$ and bulk CTPEB-Al samples, thick films were prepared and $G^{\prime}(\omega)$ and $G^{\prime \prime}(\omega)$ were measured using $2 \mathrm{~cm}$ parallel plate geometry in the $\omega$ range of $0.01 \sim 100 \mathrm{rads}^{-1}$ at seven temperatures from 15 to $75^{\circ} \mathrm{C}$. Creep and creep recovery measurements were also conducted above $C=7 \mathrm{wt} \%$ using the same rheometer.

Infrared spectra were obtained at room temperature using a FTIR spectrometer (FTS 6000, Bio-rad) with 64 scans at a resolution of $4 \mathrm{~cm}^{-1}$.

\section{RESULTS AND DISCUSSION}

\subsection{Viscosity Behavior in the Dilute Solution}

Results of viscometric measurements for dilute solutions of CTPEB-Al as well as CTPEB-Ester are shown in Fig.1 in the forms of reduced viscosity $\eta_{\mathrm{sp}} / C$ and inherent viscosity $\ln \eta_{\mathrm{r}} / C$ vs. polymer concentration $C$. For CTPEB-Ester, the reduced viscosity increases linearly with polymer concentration and its $C$ dependence is well described by the Huggins equation, $\eta_{\mathrm{sp}} / C$ $=[\eta]+k^{\prime}[\eta]^{2} C$ where $[\eta]$ is the intrinsic viscosity and $k^{\prime}$ is the Huggins constant. The value of $[\eta]$ obtained is $6.4 \mathrm{~cm}^{3} \mathrm{~g}^{-1}$.

The reduced viscosity of CTPEB-Al solutions also increased linearly with $C$ with a steep slope and extrapolation to zero concentration gave an intrinsic viscosity value of 6.88 $\mathrm{cm}^{3} \mathrm{~g}^{-1}$. The larger value of $[\eta]_{\text {CTPEB-Al }}$ compared with the $[\eta]$ of CTPEB-Ester sample implies that chains in the CTPEBAl system must be associated even at the infinite dilution 
limit owing to the strong restriction that all trivalent $\mathrm{Al}^{3+}$ ions attached to chain ends must be electrostatically neutral in nonpolar decalin and present in the form of ATC bond $\left[\left(\mathrm{COO}^{-}\right)_{3} \mathrm{Al}^{3+}\right]$. In the associating structure of CTPEB-Al, the number of $\mathrm{Al}^{3+}$ cation must be a multiple of 2 and the number of CTPEB chains must be a multiple of 3 to satisfy the electrically neutral condition of ATC bonds. The association of chains in CTPEB-Al must proceed in the following way: 3 CTPEB chains will associate with $2 \mathrm{Al}^{3+}$ cations and 6 CTPEB chains will associate with $4 \mathrm{Al}^{3+}$ cations and so on. This indicates that the smallest associating structural unit of CTPEB-Al should be made of three CTPEB chains associated to each other by two ATC bonds as shown in Fig.2. Here, the smallest associating structural unit can be present in dilute solution as any of the three forms (A, B, and C). We attempted to estimate the intrinsic viscosity of these three associating forms as follows.

Assuming that CTPEB-Ester chains obey Gaussian statistics, we express the mean-square radius of gyration of a single CTPEB-Ester chain, $\left\langle R_{\mathrm{G} 1}{ }^{2}\right\rangle$, using the average end-to-end distance $L$ of a single CTPEB-Ester chain.

$$
\left\langle R_{\mathrm{G} 1}{ }^{2}\right\rangle=\frac{1}{6} L^{2}
$$

In ignoring the interaction between the two ATC bonds, the mean-square radius of gyration $\left\langle R_{\mathrm{G}}{ }^{2}\right\rangle$ of the smallest associating structural unit of the form A in Fig. 2 is calculated as

$$
\left\langle R_{\mathrm{G}}{ }^{2}\right\rangle=\frac{1}{3} L^{2}=2\left\langle R_{G 1}{ }^{2}\right\rangle
$$

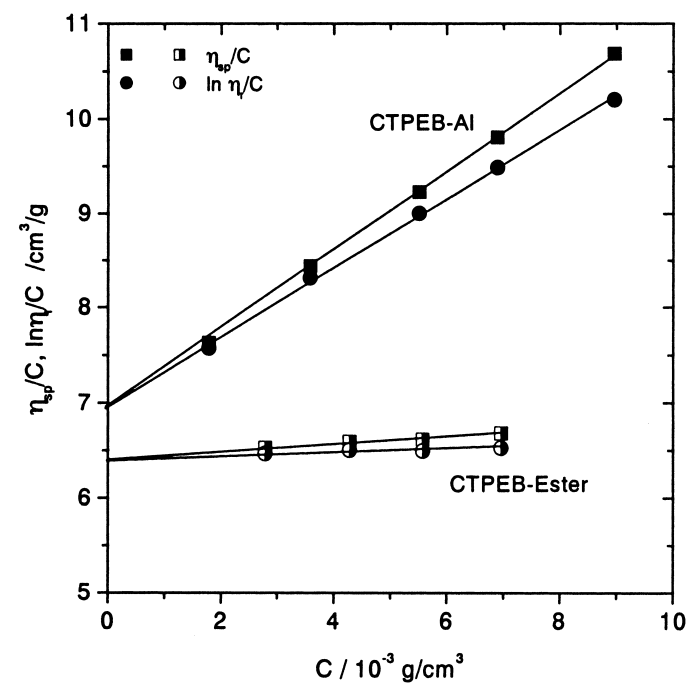

Fig.1 Concentration dependence of reduced viscosity, $\eta_{\mathrm{sp}} / C$, and inherent viscosity, $\ln \eta_{t} / C$, for CTPEB-Ester and CTPEB-Al solutions in decalin.
If we are allowed to assume that the value of the Flory viscosity constant, $\Phi$, for this associating polymer is the same as the value for the linear polymer, the relationship between intrinsic viscosity and the mean-square radius of gyration for the form A and the single CTPEB-Ester chain is given by Eqs. (3a) and (3b), respectively.

$$
\begin{aligned}
& 6^{3 / 2}\left\langle R_{\mathrm{G}}{ }^{2}\right\rangle^{3 / 2} \Phi=[\eta]_{\mathrm{A}} M \\
& 6^{3 / 2}\left\langle{R_{\mathrm{G} 1}}^{2}\right\rangle^{3 / 2} \Phi=[\eta]_{\mathrm{CTPEB}-\text { Ester }} M_{1}
\end{aligned}
$$

Here $M$ and $M_{1}$ are the molecular weights of the smallest associating unit of CTPEB-Al and the single CTPEB-Ester chain, and $[\eta]_{\mathrm{A}}$ and $[\eta]_{\mathrm{CTPEB}-\mathrm{Ester}}$ the intrinsic viscosities of the form $\mathrm{A}$ and the single CTPEB-Ester chain, respectively. Substitution of the above relations into Eqs.(1) and (2) with $M$ $=3 M_{1}$, and $[\eta]=6.4 \mathrm{~cm}^{3} / \mathrm{g}$ for CTPEB-Ester results in $[\eta]_{\mathrm{A}}=$ $6.03 \mathrm{~cm}^{3} / \mathrm{g}$, which is smaller than the experimentally obtained intrinsic viscosity value.

In the very dilution limit, attraction between the ATC bonds gives rise to contraction of the smallest associating structural unit to form a reverse micelle with three flower-like loops (the form B in Fig.2). The intrinsic viscosity of the flower-like micelle can be estimated as follows.

Mean-square end-to-end distance $\left\langle R_{\mathrm{ij}}{ }^{2}\right\rangle_{\mathrm{r}}$ between $i$ th and $j$ th segments of a loop can be written as ${ }^{42)}$

$$
\left\langle R_{\mathrm{ij}}{ }^{2}\right\rangle_{\mathrm{r}}=\mu l^{2}
$$

with $\mu=(j-i)\left(1-\frac{j-i}{n}\right)$.

Here $n$ is the number of segments in a single chain and $l$ the segment length. The radius of gyration of a micelle, $R_{\mathrm{C}}$, is easily calculated from the distance between the $j$ th segment and the centre of gravity which is located at the position of $i=$ 0 in Eq.(4), neglecting the finite size of the two ionic groups packed at the center position.

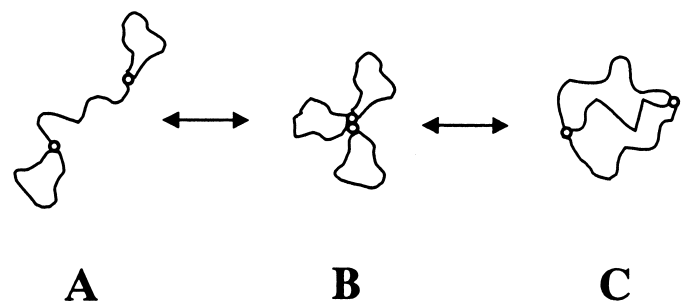

Fig.2 Schematic representation of the smallest associating structural unit of CTPEB-Al in decalin at three forms A, B, and C. The small circles represent $\mathrm{Al}^{3+}$ ions. 


$$
\left\langle R_{\mathrm{C}}{ }^{2}\right\rangle=\frac{1}{n}\left\langle\sum_{j=1}^{n} R_{0 \mathrm{j}}{ }^{2}\right\rangle \approx \frac{1}{6} n l^{2}=\left\langle R_{\mathrm{G} 1}{ }^{2}\right\rangle
$$

Thus, intrinsic viscosity of the flower-like micelle $\mathrm{B},[\eta]_{\mathrm{B}}$, is

$$
[\eta]_{\mathrm{B}}=6^{3 / 2} \Phi \frac{\left\langle R_{\mathrm{C}}{ }^{2}\right\rangle^{3 / 2}}{M}=6^{3 / 2} \Phi \frac{\left\langle R_{\mathrm{G} 1}{ }^{2}\right\rangle^{3 / 2}}{3 M_{1}}=\frac{[\eta]_{\text {CTPEB-Ester }}}{3}
$$

Putting the intrinsic viscosity value of CTPEB-Ester in Eq.(5) gives $[\eta]_{\mathrm{B}}=2.14 \mathrm{~cm}^{3} / \mathrm{g}$ which is much smaller than the experimentally obtained $[\eta]_{\text {CTPEB-Al }}=6.88 \mathrm{~cm}^{3} / \mathrm{g}$. The meansquare radius of gyration for the form $\mathrm{C}$ of the smallest associating structural unit can be shown to be larger than that of CTPEB-Ester by a factor of $4 / 3$, thus $[\eta]_{\mathrm{C}}=2.84 \mathrm{~cm}^{3} / \mathrm{g}$. Among three forms of the smallest associating unit of CTPEB$\mathrm{Al}$, the form A gives $[\eta]$ close to but smaller than the experimental value in the infinite dilution limit, while other two forms give much smaller values. This suggests that, even in the infinite dilution limit, a fraction of primary CTPEB chains forms aggregates with a size larger than the smallest unit and further association may occur with increasing $C$, judging from the $C$ dependence of $\eta_{\mathrm{sp}} / C$ in Fig.1. It is to be noted that the next larger associated structure composed of 6 chains and 4 ATC bonds gives $[\eta]=12.7 \mathrm{~cm}^{3} / \mathrm{g}$ in the most extended form.

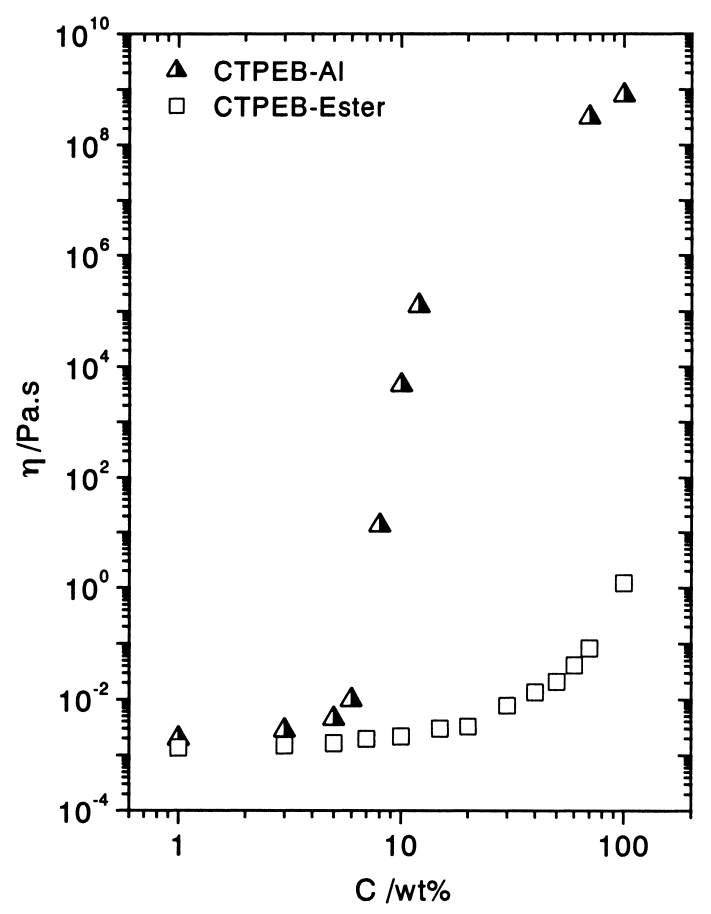

Fig.3 Concentration dependence of viscosity for CTPEB-Al and CTPEB-Ester solutions in decalin and in the bulk at $55^{\circ} \mathrm{C}$.

\subsection{Viscosity Behavior above $C=1 \mathrm{wt} \%$}

The concentration dependence of steady viscosity $\eta$ for CTPEB-Ester and CTPEB-Al samples at $55^{\circ} \mathrm{C}$ is shown in Fig.3. The $\eta$ of the CTPEB-Al solutions was obtained up to $C$ $=7 \mathrm{wt} \%$ by the steady flow measurement. Above $C=8 \mathrm{wt} \%$, $\eta$ was estimated from the creep and creep recovery data. The $\eta$ of CTPEB-Al solutions is higher but comparatively close to that of the CTPEB-Ester solutions up to $C=5 \mathrm{wt} \%$. Above $C$ $=6 \mathrm{wt} \%, \eta$ of CTPEB-Al solutions increased much more rapidly with a steep slope up to $C=12 \mathrm{wt} \%$ and the $\eta$ value for bulk CTPEB-Al reached about $10^{9}$ Pas which is about $10^{9}$ times larger than the $\eta$ value of bulk CTPEB-Ester. Total increase in $\eta$ for CTPEB-Ester solutions from $C=1$ to 100 $\mathrm{wt} \%$ is found to be around 3 orders of magnitude, which is attributable to an increase in the segmental friction coefficient with increasing $C$. As mentioned elsewhere, ${ }^{40)}$ CTPEB-Ester chains are not entangled even in the bulk state.

As stated above, most of CTPEB-Al chains are associated with each other even at the infinite dilution limit in the form of smallest associating structural unit made of three CTPEB chains. With increasing concentration, association among these smallest associating structural units proceeds so that larger polymeric clusters are formed. The viscosity increment up to $C=6 \mathrm{wt} \%$ should be due to the formation of large polymer

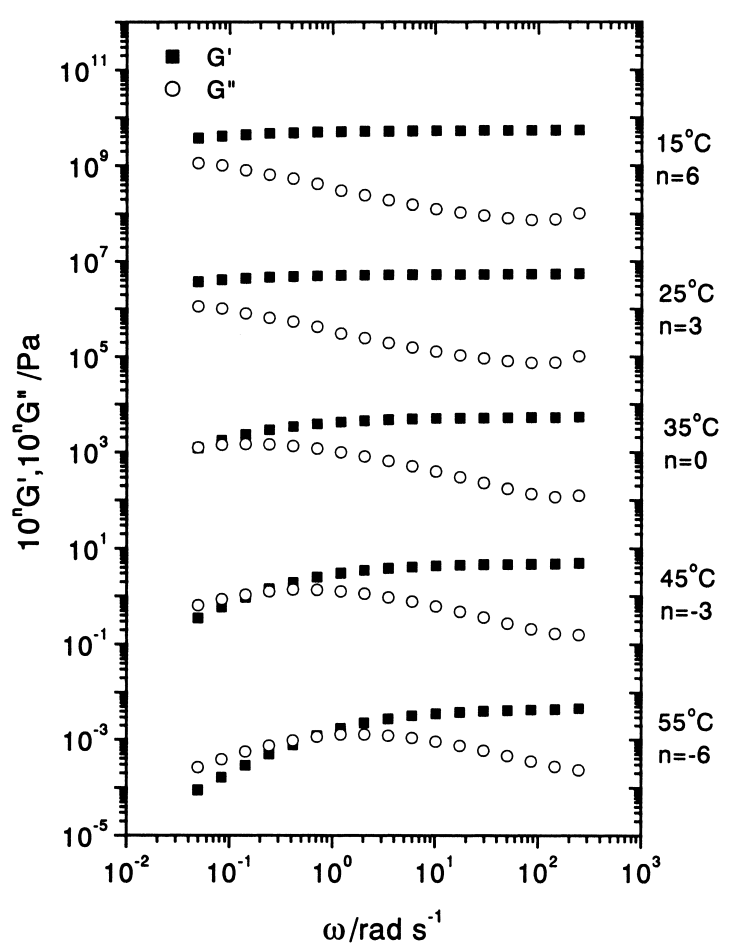

Fig.4 Angular frequency $\omega$ dependencies of $G^{\prime}(\omega)$ and $G^{\prime \prime}(\omega)$ for the $10 \mathrm{wt} \%$ CTPEB-Al solution in decalin at five temperatures from 15 to $55^{\circ} \mathrm{C}$. The curves are shifted by $10^{\mathrm{n}}$ for clarity of the figure. 
clusters of various sizes in the samples. As concentration further increases, these polymeric clusters made of the smallest associating unit come close to each other so that a viscoelastic network is formed mainly via ionic crosslinks. Formation of a network extending over the whole system should have occurred at around $C=7 \mathrm{wt} \%$. Thus, $\eta$ at $C=8$ $\mathrm{wt} \%$ increased 3 orders of magnitude over $\eta$ at $C=6 \mathrm{wt} \%$. The network becomes stronger as $C$ increases, nevertheless they can rearrange by recombination of associating points in ionic clusters as shall be discussed later, so that finite viscosity is observed even for the bulk sample at a higher temperature of $75^{\circ} \mathrm{C}$.

\subsection{Viscoelasticity of CTPEB-Al Solutions above $C=$ $7 \mathrm{wt} \%$}

Figure 4 shows dynamic viscoelastic responses of the $10 \mathrm{wt} \%$ CTPEB-Al solution in decalin in the $T$ range of 15 $55^{\circ} \mathrm{C}$. At $15^{\circ} \mathrm{C}$, the solution behaves as an elastic network characterised by $\omega$-independent $G^{\prime}(\omega)$ as well as $G^{\prime \prime}(\omega)$ much smaller than $\mathrm{G}^{\prime}(\omega)$ in the whole frequency region. At $55^{\circ} \mathrm{C}$, $\mathrm{G}^{\prime \prime}(\omega)$ becomes larger than $\mathrm{G}^{\prime}(\omega)$ in the low frequency region, which indicates that the solution starts to flow in the long time region. The $\mathrm{G}^{\prime}(\omega)$ and $\mathrm{G}^{\prime \prime}(\omega)$ curves were superposed to obtain

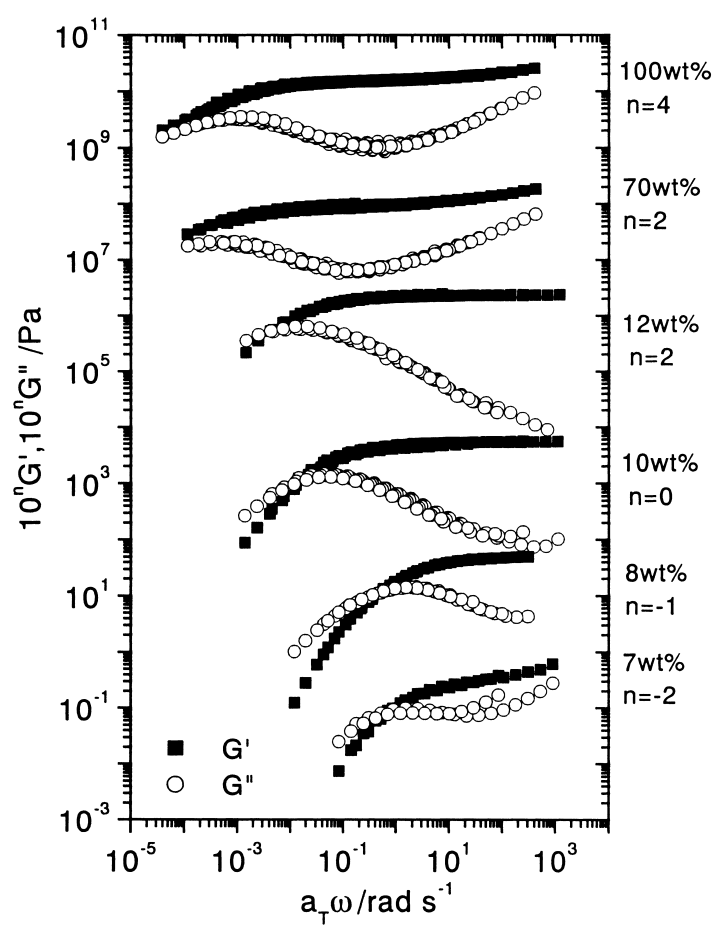

Fig.5 Master curves obtained from the time-temperature reduction scheme for CTPEB-Al samples in decalin with different concentrations from $7 \mathrm{wt} \%$ (the bottom) to $100 \mathrm{wt} \%$ (the top). The reference temperature $T_{\mathrm{r}}$ is taken $25^{\circ} \mathrm{C}$. The curves are shifted by $10^{\mathrm{n}}$ as indicated. a master curve by shifting horizontally by the shift factor $a_{\mathrm{T}}$. The reference temperature $T_{\mathrm{r}}$ was chosen $25^{\circ} \mathrm{C}$. Results shown in Fig. 5 show that the time-temperature reduction scheme is applicable for this solution, and the scheme was also found to be applicable for other solutions with $C \geq 8 \mathrm{wt} \%$ as shown in the same figure. The success of the time-temperature reduction scheme indicates that the relaxation mechanisms of these viscoelastic networks are not influenced by thermal agitation in the $T$ range studied. We succeeded to measure the dynamic vicsoelasticity of $C=7 \mathrm{wt} \%$ solution at 15 and $25^{\circ} \mathrm{C}$ but their superposition was not good especially in the high frequency region. A rise of $G^{\prime \prime}(\omega)$ in the higher $\omega$ region may be related to molecular motions of long branched chains inside huge clusters which are connected to each other with a very small number of junction points as evidenced from the ratio of the number density of the elastically effective chains to that of primary chains shown in Fig.8. In the dynamic vicsoelastic data of $C=70$ and $100 \mathrm{wt} \%$ samples, two relaxation modes are seen. One is in the high frequency region and the other in the low frequency regime. These two relaxation modes should be related to dissociation of ionic associating points and will be discussed later in this section.

Figure 6 shows a semilogarithmic plot of the horizontal shift factor $a_{\mathrm{T}}$ for $8 \sim 100 \mathrm{wt} \%$ CTPEB-Al samples and the viscosity ratio $T_{\mathrm{r}} \eta(T) / T \eta\left(T_{\mathrm{r}}\right)$ for $1 \sim 7 \mathrm{wt} \%$ solutions against $1 / T$. Here,

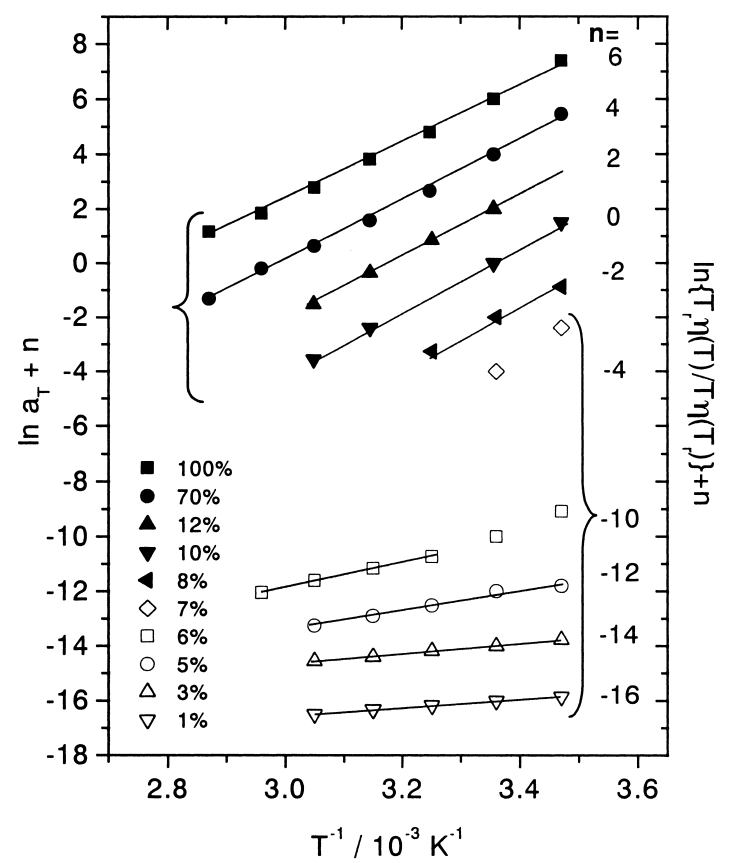

Fig.6 Semilogarithmic plot of shift factor, $a_{\mathrm{T}}$, against $1 / T$ for CTPEBAl samples with $C=8 \sim 100 \mathrm{wt} \%$. Viscosity ratio $T_{\mathrm{r}} \eta(T) / T \eta\left(T_{\mathrm{r}}\right)$ is also plotted for CTPEB-Al solutions with $C=1 \sim 7 \mathrm{wt} \%$. The data are shifted by $10^{\mathrm{n}}$ for clarity of the figure. 
the latter value should take the same value as the corresponding shift factor $a_{\mathrm{T}}$, if they could be measured, because the timetemperature superposition principle was presumably satisfied for these samples. Figure 6 clearly shows that the temperature dependence is well described by the Arrhenius type of equation:

$$
a_{\mathrm{T}} \sim \exp \left(\frac{H}{R T}\right)
$$

where $H$ is the activation energy and $R$ is the universal gas constant. From slopes of the straight lines in Fig.6, we estimated $H$ for samples with different concentration, which are listed in Table I. Semilogarithmic plots of the viscosity ratio $T_{\mathrm{r}} \eta(T) / T \eta\left(T_{\mathrm{r}}\right)$ against $1 / \mathrm{T}$ for CTPEB-Ester solutions with various concentrations $C$ in the range of $1-100 \mathrm{wt} \%$, though not shown in the figure, were also found to follow the Arrhenius type of equation very well. Values of the activation energy calculated are also listed in Table I. The table shows that $H$ monotonously increases with increasing $C$ for the CTPEB-Ester system to an experimental accuracy, which results in enhancement in the relaxation time $\tau$. Values of $H$ of CTPEB-Al samples increase with increasing $C$ upto $C=12$ $\mathrm{wt} \%$, but appear to slightly decrease at higher $C$ of 70 and 100 $\mathrm{wt} \%$.

The $C$ dependence of $\tau$ can be discussed based on the consideration that $\tau$ is conveniently expressed by the product

Table I Activation energy, $H$, for CTPEB-Al and CTPEB-Ester at different concentrations from 1 to $100 \mathrm{wt} \%{ }^{\mathrm{a}}$

\begin{tabular}{|c|c|c|}
\hline \multirow{2}{*}{$\begin{array}{c}\text { Concentration } \\
/ \mathrm{wt} \%\end{array}$} & \multicolumn{2}{|c|}{$H / \mathrm{kJmol}^{-1}$} \\
\cline { 2 - 3 } & CTPEB-Al & CTPEB-Ester \\
\hline 100 & 89 & 67 \\
\hline 70 & 91 & 38 \\
\hline 50 & - & 33 \\
\hline 40 & - & 25 \\
\hline 30 & - & 21 \\
\hline 20 & - & 18 \\
\hline 15 & - & 17 \\
\hline 12 & 104 & 17 \\
\hline 10 & 99 & 16 \\
\hline 8 & 90 & 16 \\
\hline 7 & - & 16 \\
\hline 6 & 37 & 15 \\
\hline 5 & 30 & 14 \\
\hline 3 & 15 & 14 \\
\hline 1 & 13 & 13 \\
\hline
\end{tabular}

${ }^{\text {a }}$ Experimental errors lie between $\pm 0.5 \mathrm{kJmol}^{-1}$ for both systems. of the two contributions, one of which is the local friction factor $\zeta(C, T)$ of the segment or the single CTPEB main chain with low molecular weight and another the global structure factor $\psi(C, T)$. The contribution to $H$ from $\zeta(C, T)$ at each $C$ can be estimated from $H$ of the corresponding CTPEB-Ester sample, since it has no specific intermolecular interaction at chain ends and remains a viscous fluid in the bulk. Then the contribution to $H$ from the structure factor is estimated as the activation energy difference $\Delta H$ between CTPEB-Al and CTPEB-Ester, which is plotted against $C$ in Fig.7. Corresponding to the steady state viscosity behavior in Fig.3, there occurs a remarkable increase of $\Delta H \approx 74 \mathrm{kJmol}^{-1}$ in the narrow $C$ range of $3 \sim 8 \mathrm{wt} \%$. The $\Delta H$ value increases up to $C=$ $12 \mathrm{wt} \%$, then decreases with increasing $C$. The value of $\Delta H$ in bulk was found to be as small as about $22 \mathrm{kJmol}^{-1}$.

The changes of $\Delta H$ with $C$ may be related to changes in the relaxation mechanisms of the solutions. At and below $6 \mathrm{wt} \%$, the mechanical relaxation should take place by flow of a suspension of the clusters formed by association of polymers, where the relaxation is mainly dominated by mobility of the solvent, so that the observed $\Delta H$ is fairly small. When the cluster size increases and an occupation ratio of space by clusters approaches to unity, a viscoelastic network will be formed. Relaxation of this network occurs via detachment of associating points. An activation energy for detachment of associating points must be much higher than that needed for the polymer clusters to flow in the solvent, which explains higher $\Delta H$ for samples with $C \geq 7 \mathrm{wt} \%$ in comparison with $\Delta H$ for the samples with $C$ less than $6 \mathrm{wt} \%$. The large decrease of $\Delta H$ for $C=70$ and $100 \mathrm{wt} \%$ samples may be related to

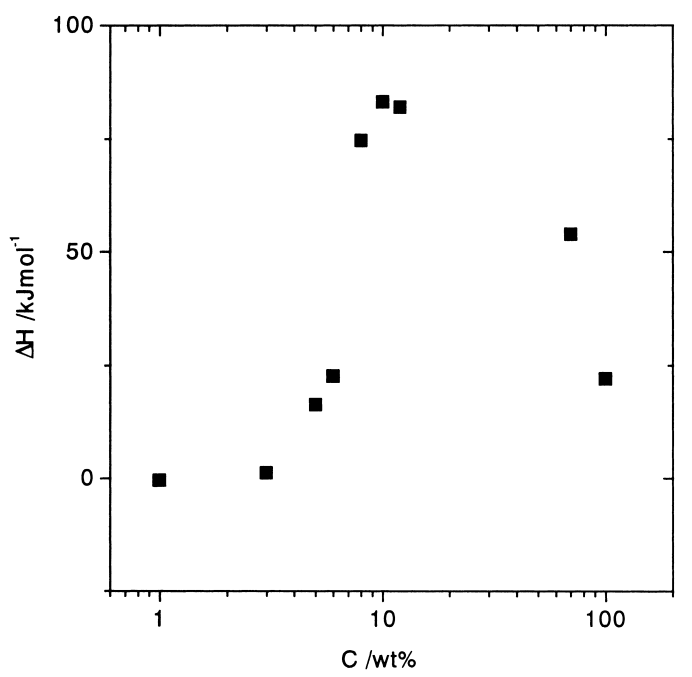

Fig.7 Concentration dependence of activation energy difference $\Delta H$ between CTPEB-Al and CTPEB-Ester solutions. 
appearance of the fast relaxation mode which occurs due to dissociation of weaker associating points. As will be revealed from the IR spectroscopic data discussed in the following section, different types of associating points are present in the CTPEB-Al networks. One is the single ATC bond, which can act as an effective crosslinking point only if the bond is composed of three CTPEB chains and one $\mathrm{Al}^{3+}$ cation without a loop. In this case, detachment of chains from the single ATC bond is almost impossible in such a nonpolar solvent as decalin. Ion clusters made of a number of ATC bonds can also act as effective crosslinks. However ion clusters made of ATC bonds can be present either with or without loops. Ion clusters with loops can dissociate more readily than ion clusters without loops, because in the former case network relaxation can takes place by the escape of an ATC bond together with a chain and a loop from ion clusters, while the latter case needs simultaneous relaxation of three network chains due to trivalent nature of the $\mathrm{Al}^{3+}$ cation. Thus the fast relaxation mode present at $C=70$ and $100 \mathrm{wt} \%$ CTPEB-Al samples may be due to the dissociation of the single ATC bond with a loop from ion clusters. The dissociated ATC bond migrates in the network, re-enter another ion cluster, and then the loop is once again used for relaxation of the whole network. This may explain broadening of relaxation time distributions as well as the rather small value of $\Delta H$ at higher $C$.

We calculated the ratio of the number density of the elastically effective chains, $v_{\text {eff, }}$, to that of primary chains, $v$, at $25^{\circ} \mathrm{C}$ using the equation $G_{\text {plat }}=v_{\text {eff }} k_{\mathrm{B}} T$ where $G_{\text {plat }}$ is the high frequency plateau modulus of $G^{\prime}$ observed for the solutions with $C \geq 7 \mathrm{wt} \%$ and the front factor was assumed unity. As

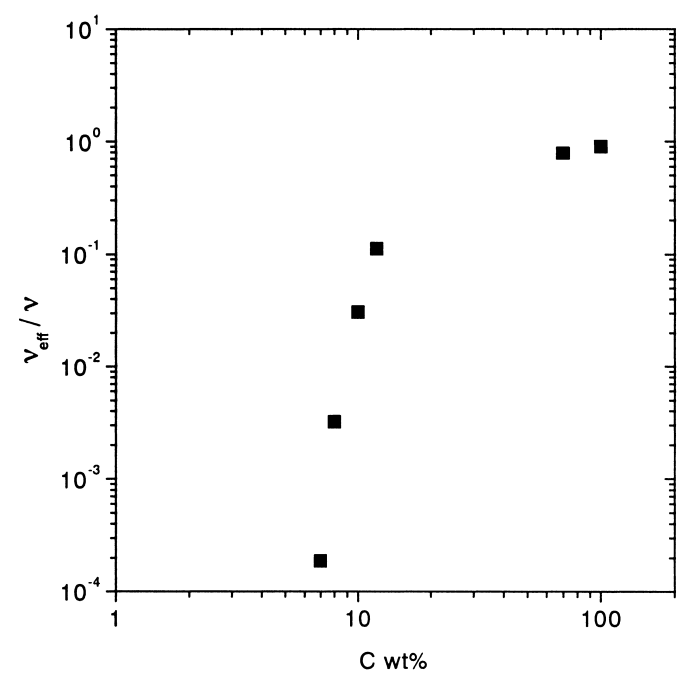

Fig.8 Concentration dependence of a ratio of the number of elastically effective chains to that of total chains for $C=7 \sim 100 \mathrm{wt} \%$. shown in Fig. 8, the value of $v_{\text {eff }} / v$ is very small at $C=7 \mathrm{wt} \%$ and gradually increases with increasing $C$. The value of $v_{\text {eff }} / v$ for $C=12 \mathrm{wt} \%$ is 0.11 and increases up to 0.89 in the bulk. Thus it is reasonable to suppose that in the low concentration range from $7 \mathrm{wt} \%$ to $12 \mathrm{wt} \%$, CTPEB-Al chains are associated with each other in such a way that they form pretty long network strands with or without branches as dangling chains, which act as elastically effective chains as a whole. When an ATC bond belonging to branched chains happens to touch to an ATC bond participating in formation of main elastically effective chains, exchange of a loop between the ATC bonds induces relaxation of the elastically effective network strand. In the higher concentration samples of $C=70$ and $100 \mathrm{wt} \%$, most of the ATC bonds participate in network formation as effective crosslinks, while loops are mainly present in ion clusters. The IR spectroscopy is useful to estimate the relative ratio of single ATC bonds and ionic clusters made of ATC bonds.

\subsection{IR Spectra of CTPEB-AI}

The IR spectra of the CTPEB-Al system showed a broad absorption band of the carbonyl group in the wave number range of $1555-1685 \mathrm{~cm}^{-1}$ to be assigned as the stretching mode for all concentrations from $3 \sim 100 \mathrm{wt} \%$ as shown in Fig.9.

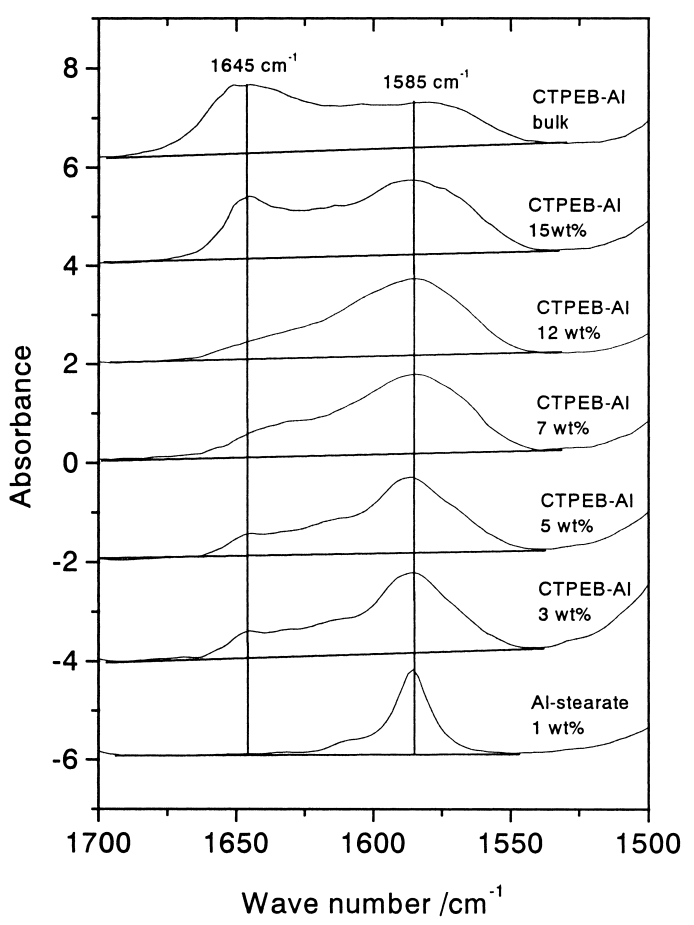

Fig.9 IR spectra of CTPEB-Al solutions in the range of $C$ from 3 to 100 $\mathrm{wt} \%$ in decalin. IR spectrum of $C=1 \mathrm{wt} \% \mathrm{Al}$-stearate in decalin is also shown at the bottom. 
Though this mode appears to consist of a couple of overlapped peaks, we picked up two bands at $1585 \mathrm{~cm}^{-1}$ and $1645 \mathrm{~cm}^{-1}$ as absorbances from the single ATC bond and ionic clusters. To determine which band is originated from the single ATC bond, we measured the IR spectra of aluminium stearate (Alstearate) samples in the $C$ range from $1 \mathrm{wt} \%$ in decalin to the bulk. The spectrum of $1 \mathrm{wt} \% \mathrm{Al}$-stearate in decalin is shown in Fig.9 for comparison. Other samples also gave a single band at $1585 \mathrm{~cm}^{-1}$ and there was no band at $1645 \mathrm{~cm}^{-1}$ in Al-stearate samples. Thus we consider that the band at $1585 \mathrm{~cm}^{-1}$ in the CTPEB-Al samples is attributable to the single ATC bond, while the band at $1645 \mathrm{~cm}^{-1}$ may be from the ionic clusters.

If the peak intensity of carbonyl groups is assumed to be the same irrespective of whether they are located inside the ionic cluster or in the single ATC bond, the relative area of IR bands would represent the relative number of $\mathrm{Al}^{3+}$ cations in the two states. However, we could not estimate the relative area of the two bands accurately. Assuming that the relative height of respective bands at $1585 \mathrm{~cm}^{-1}$ and $1645 \mathrm{~cm}^{-1}$ roughly represent their relative area, we estimated the relative number of aluminium cations in single ATC bonds and in ion clusters. Figure 10 indicates that below $C=12 \mathrm{wt} \%$ the relative number of single ATC bonds is much larger than the relative number of $\mathrm{Al}^{3+}$ cation in ionic clusters, and the relative number of ATC bonds even slightly increases with increasing $C$ from 0.75 to 0.8 up to $C=12 \mathrm{wt} \%$. Above $C=15 \mathrm{wt} \%$ the relative number of single ATC bonds significantly decreases and its value reaches to 0.4 for the bulk CTPEB-Al. Thus in the bulk sample, about $60 \% \mathrm{Al}^{3+}$ cations are located in ionic clusters, while about $40 \% \mathrm{Al}^{3+}$ cations are present as single ATC bonds.

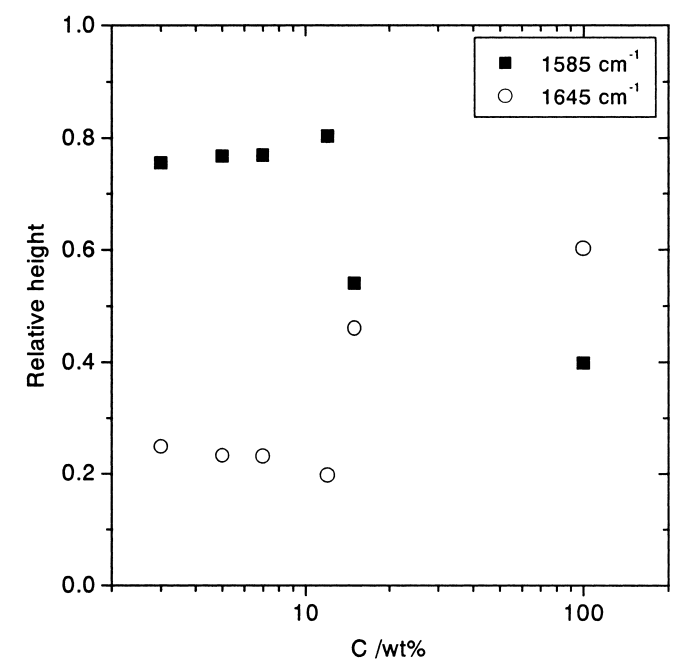

Fig.10 Variation of relative height of bands at 1585 and $1645 \mathrm{~cm}^{-1}$ with concentration.

\section{Acknowledgement}

The authors are grateful to Nippon Soda Co. Ltd. for a gift of CTPEB samples.

\section{REFERENCES}

1) Eisenberg A, Kim J-s, Introduction to Ionomers, Wiley Interscience: New York (1998).

2) Tant MR, Mauritz KA, Wilkes GL, Ionomers: Synthesis, Structure, Properties and Applications, Blackie Academic \& Professional, New York (1997).

3) Schlick S, Ionomers: Charactreization, Theory, and Application, CRC Press Inc, New York (1996).

4) Eisenberg A, King M, Ion-Containing Polymers, polymer physics, Stein RS, Ed, Academic Press, New York, Vol. 2 (1977).

5) Fitzgerald JJ, Weiss RA, J Macromol Sci, Rev Macromol Chem Phys, C28, 99 (1988).

6) Johannsson R, Chassenieux C, Durand D, Nicolai T, Vanhoorne P, Jerome R, Macromolecules, 28, 8504 (1995).

7) Chassenieux C, Nicolai T, Tassin JF, Durand D, Gohy JF, Jerome R, Macromol Rapid Commun, 22, 1216 (2001).

8) Chassenieux C, Tassin JF, Gohy JF, Jerome R, Macromolecules, 33, 1796 (2000).

9) Chassenieux C, Johannsson R, Durand D, Nicolai T, Vanhoorne P, Jerome R, Colloids and Surfaces, 112, 155 (1996).

10) Nomula S, Cooper SL, Macromolecules, 34, 925 (2001).

11) Nomula S, Cooper SL, Macromolecules, 34, 2653 (2001).

12) Nomula S, Cooper SL, Macromolecules, 33, 6402 (2000).

13) Karayianni E, Jerome R, Cooper SL, Macromolecules, 28, 6494 (1995).

14) Karayianni E, Jerome R, Cooper SL, Macromolecules, 33, 6473 (2000).

15) Jalal V, Duplessix R, J Phys France, 49, 1775 (1988).

16) Ma X, Sauer JA, Hara M, Macromolecules, 28, 5526 (1995).

17) Duchesne D, Eisenberg A, Can J Chem, 1990, 68, 1228.

18) Vanhoorne P, Van den Bossche G, Fontaine F, Sobry R, Jerome R, Stamm M, Macromolecules, 27, 838 (1994).

19) Ehmann M, Galin J-C, Polymer, 33, 859 (1992).

20) Broze G, Jerome R, Teyssie P, J Polym Sci, 21, 2205 (1983).

21) Broze G, Jerome R, Teyssie P, Marco C, Macromolecules, 16, 1771 (1983).

22) Broze G, Jerome R, Teyssie P, Marco C, Macromolecules, 18, 1376 (1985).

23) Tant MR, Wilkes GL, Kennedy JP, J Appl Polym Sci, 42, 523 (1991).

24) Tant MR, Wilkes GL, Kennedy JP, J Appl Polym Sci, 37, 2873 (1989).

25) Tant MR, Wilkes GL, Read MD, Kennedy JP, J Polym Sci, 24, 619 (1986). 
26) Fan X-D, Bazuin CG, Macromolecules, 26, 2508 (1993).

27) Quiram DJ, Register RA, Ryan AJ, Macromolecules, 31, 1432 (1998).

28) Chu B, Wu DQ, Lundberg RD, MacKnight WJ, Macromolecules, 26, 994 (1993).

29) Sauer BB, McLean RS, Macromolecules, 33, 7939 (2000).

30) Kutsumizu S, Tagawa H, Muroga Y, Yano S, Macromolecules, 33, 3818 (2000).

31) Kutsumizu S, Goto M, Yano S, Schlick S, Macromolecules, 35, 6298 (2002).

32) Lantman CW, MacKnight WJ, Higgins JS, Peiffer DG, Sinha SK, Lundberg RD, Macromolecules, 21, 1339 (1988).

33) Aldebert P, Dreyfus B, Pineri M, Macromolecules, 19, 2651 (1986).

34) Hara M, Wu J, Macromolecules, 21, 402 (1988).
35) Gotoh Y, Imakita J, Ohkoshi Y, Nagura M, Polym J, 32-10, 838 (2000).

36) Kutsumizu S, Hara H, Tachino H, Shimabayashi K, Yano S, Macromolecules, 32, 6340 (1999).

37) Coleman MM, Lee JY, Painter PC, Macromolecules, 23, 2339 (1990).

38) Painter PC, Brozoski BA, Coleman MM, J Polym Sci Polym Phys, 20, 1069 (1982).

39) Kitao H, Takada A, Nemoto N, Journal of the Society of Rheology Japan, 25, 201 (1997)

40) Rahman SA, Takada A, Nemoto N, Journal of the Society of Rheology Japan, 30-2, 95 (2002).

41) Rahman SA, Kitao H, Nemoto N, submitted to Macromolecules.

42) Yamakawa H, Modern Theory of Polymer Solutions, Harper \& Row, New York (1971). 
